\title{
Instrumental variable methods in clinical research
}

\author{
Emilia Bagiella, PhD, ${ }^{\mathrm{a}}$ Tara Karamlou, MD, ${ }^{\mathrm{b}}$ Helena Chang, MS, ${ }^{\mathrm{a}}$ and John Spivack, $\mathrm{PhD}{ }^{\mathrm{a}}$
}

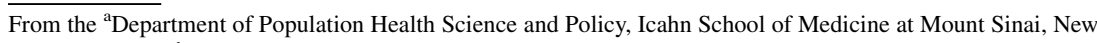
York, NY; and ${ }^{\mathrm{b}}$ Division of Pediatric Cardiothoracic Surgery, UCSF School of Medicine, San Francisco, Calif. This work was partially supported by a cooperative agreement (U01 HL088942) funded by the National Heart, Lung, and Blood Institute.

Received for publication July 17, 2015; accepted for publication July 17, 2015; available ahead of print Aug 13, 2015.

Address for reprints: Emilia Bagiella, PhD, Department of Populations Health Science and Policy, Icahn School of Medicine at Mount Sinai, One Gustave L. Levy Place, Box 1077, New York, NY 10029 (E-mail: emilia. bagiella@mountsinai.org).

J Thorac Cardiovasc Surg 2015;150:779-82 $0022-5223 / \$ 36.00$

Copyright (c) 2015 by The American Association for Thoracic Surgery http://dx.doi.org/10.1016/j.jtcvs.2015.07.056
}

Randomized trials are the most valid approach to assess the causal relationship between an exposure and an outcome of interest. Randomization ensures that the experimental groups being compared are similar in terms of observed and unobserved baseline and prognostic characteristics. In nonrandomized studies, selection and allocation bias arise as the result of individuals' or physicians' choice of treatment. These biases are insidious and often difficult to identify and can have devastating effects on the inferential process. However, the availability of clinical data from national registries, large administrative databases, and electronic medical records stimulates research questions that may not be feasibly answered by a randomized clinical trial because randomization is not ethically possible, investigators lack equipoise, or prohibitively large trials would be required. Questions such as "Do patients who receive percutaneous coronary intervention (PCI) after a myocardial infarction (MI) live longer than patients who are treated pharmacologically?" could be answered using existing data. The most challenging issue in analyzing these kind of data is, of course, the comparability of the risk groups. Patients with MI who receive PCI, for example, may have more severe disease than patients who do not receive surgery and therefore may be at higher risk for an adverse outcome. On the other hand, patients who do not receive PCI may present with specific conditions that prevent them from undergoing catheterization. Therefore, determining which approach is more effective without properly accounting for differences between patient groups is problematic.

Correction of these imbalances through statistical modeling (eg, multivariable models) is not effective when important covariates are not available, there is an extreme imbalance of the background characteristics, or an overly complex regression model would be required. In addition, the resulting regression parameters could still be biased if important factors are not included in the model.

Alternatively, 2 statistical approaches can be used to eliminate or reduce selection and allocation bias: propensity

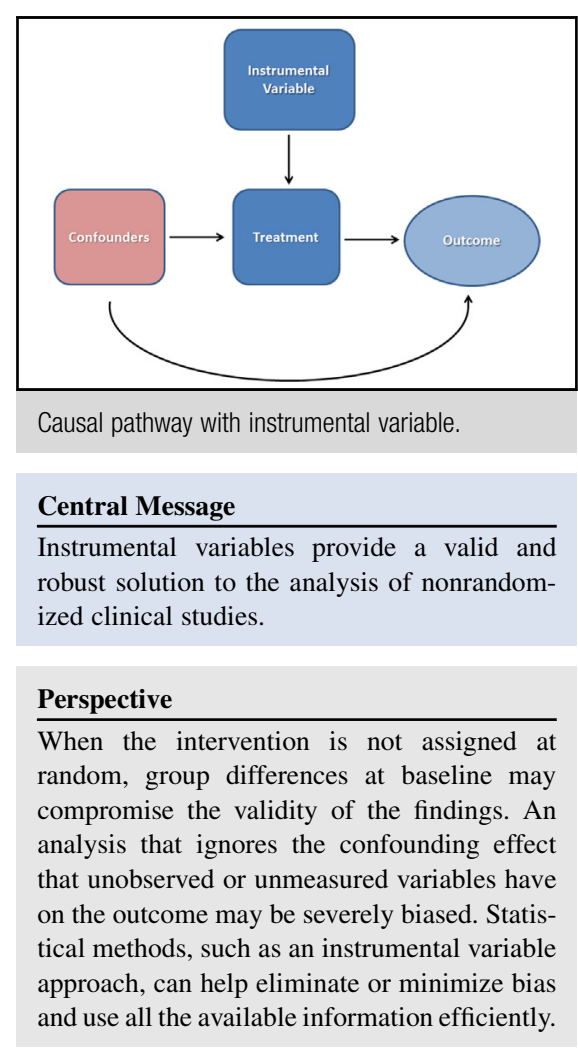

scores and instrumental variables. Both methods aim, in different ways, at producing comparable groups with respect to important characteristics, almost in the same way a randomization process would. When important confounding variables are not known or observed, an instrumental variable approach is more appropriate than propensity scores.

\section{INSTRUMENTAL VARIABLE ANALYSIS}

The principle behind instrumental variable analysis is that of identifying a variable $(Z)$, which we call the instrument, that is associated with the exposure (or treatment) of interest (B) but not directly with the outcome (C) (Figure 1). Therefore, the instrument $(\mathrm{Z})$ is related to the outcome (C) only through the exposure of interest (B).

Consider the path diagram in Figure 1 where each arrow represents a "causal" link (ie, a mechanism to generate values, not simply a correlation from other factors in common). Our interest is in the relationship between the exposure, or treatment (eg, whether or not a patient receives PCI), and an outcome such as mortality. When the treatment is not assigned at random, other factors (A), such as severity of disease, influence the outcome, both directly and indirectly, and 


\section{Abbreviations and Acronyms \\ MI = myocardial infarction \\ $\mathrm{PCI}=$ percutaneous coronary intervention}

the choice of treatment. In such circumstances, the treatment or exposure of interest is subject to some of the same influences as the response variable and is usually referred to as an "endogenous variable." Thus, if we simply proceeded to determine the relationship between treatment and outcome, (B) and (C), ignoring other factors we would obtain a biased estimate of the effect of the exposure. For example, we could attribute a more favorable outcome to the pharmacologic treatment of MI when, in fact, less severe disease in patients treated pharmacologically is the true predictor for their increased survival. The role of the instrument $\mathrm{Z}$ is to eliminate the effect of the confounders A.

In the simple linear regression case with a continuous outcome variable (eg, a quality of life scale or a biological variable), the relationships in Figure 1 can be expressed as

$$
\begin{aligned}
& C=\mu_{c}+\beta_{C A} A+\beta_{C B} B+\varepsilon_{C} \\
& B=\mu_{B}+\beta_{B A} A+\beta_{B Z} Z+\varepsilon_{B}
\end{aligned}
$$

where $\mu_{B}$ and $\mu_{c}$ represent the means of $\mathrm{B}$ and $\mathrm{C}$, respectively, and $\varepsilon_{\mathrm{B}}$ and $\varepsilon_{\mathrm{C}}$ are mutually independent error terms with mean zero. Model 1 captures the relationship between the exposure $\mathrm{B}$ and the outcome $\mathrm{C}$ controlling for the confounder A. Equation (2) models the relationship among the confounder, instrument, and exposure. The objective is to estimate the causal effect of $\mathrm{B}$ on $\mathrm{C}, \beta_{C B}$. As noted, simple regression of $\mathrm{C}$ on $\mathrm{B}$ produces a biased estimate of this effect because of the additional connection (backdoor path) between A and C. Specifically, the correlation between variables B and C does not correctly capture the causal effect because of the additional contribution to the correlation through the "confounder" A. Such confounding (endogeneity) problems also can be expressed as a correlation between the error terms $\varepsilon_{\mathrm{C}}$ and $\varepsilon_{\mathrm{B}}$.

Note that the first model would be adequate and direct estimation of $\beta_{C B}$ adjusting for A would correctly estimate the relationship between $\mathrm{B}$ and $\mathrm{C}$ if $\mathrm{A}$ were known and measurable. However, as we noted, potential confounders are often unknown or unmeasured, making the model inadequate. A solution is to introduce an instrumental variable, $\mathrm{Z}$, which affects the outcome $\mathrm{C}$ only through its influence on $\mathrm{B}$ (in the same way a randomization assignment would influence the outcome only through the treatment assigned). The role of the instrumental variable is to introduce a disturbance in B without any influence on the confounding variables or any direct influence on C. This independent disturbance of $\mathrm{B}$ allows estimation of the

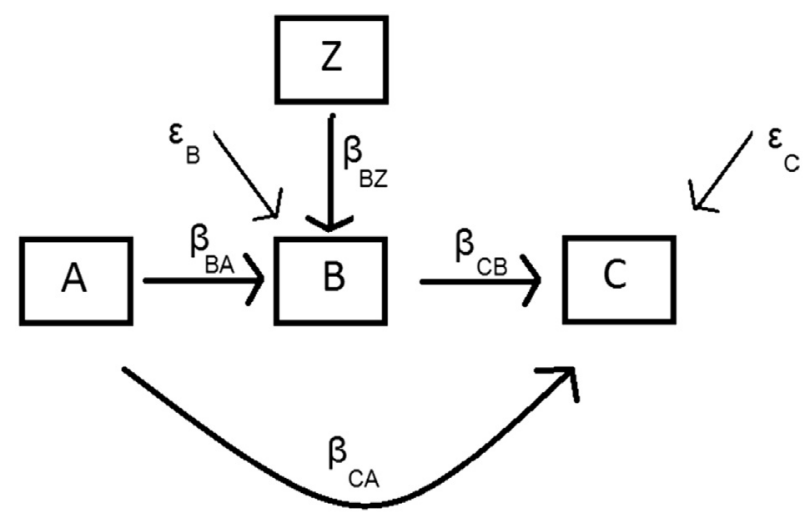

FIGURE 1. Causal pathway with instrumental variable.

effect of B on C; heuristically, it generates a randomization. In regression terms, we may write the influence of the instrument $\mathrm{Z}$ on the outcome $\mathrm{C}$ as the product of influences: $\beta_{C Z}=\beta_{B Z} \times \beta_{C B}$ or $\beta_{C B}=\beta_{C Z} / \beta_{B Z}$. Both numerator and denominator of this expression can be estimated by regression without confounding. The "instrumental" estimator $\hat{\beta}_{C B_{-} I}$ is then $\hat{\beta}_{C Z} / \hat{\beta}_{B Z}$, with $\hat{\beta}_{C Z}$ and $\hat{\beta}_{B Z}$ the regression estimates of the true coefficients $\beta_{C Z}$ and $\beta_{B Z}$. In practice, $\hat{\beta}_{C B_{-} I}$ and its correct standard error are calculated by the 2 -stage least-squares procedure. The analysis consists of 2 models:

$$
\begin{aligned}
& B=\mu_{B}+\beta_{B Z} Z+\varepsilon_{\mathrm{B}} \\
& C=\mu_{c}+\beta_{C B} B+\varepsilon_{\mathrm{C}}
\end{aligned}
$$

A first regression of $\mathrm{B}$ on $\mathrm{Z}$ would obtain predicted values of $\mathrm{B}$, for each subject, free of confounding. The second stage would then regress $C$ on these predictions, yielding $\hat{\beta}_{C B_{-} I}$. Of note, the variance of $\hat{\beta}_{C B_{-} I}$ is larger than that reported from the second-stage regression alone, and a different formula is used.

\section{UNDERLYING ASSUMPTIONS OF INSTRUMENTAL VARIABLE ANALYSIS}

A correct instrumental variable analysis rests on 3 fundamental assumptions:

(1) The instrument must be correlated with the exposure of interest (relevance).

(2) The instrument must be independent of the confounder (exogeneity).

(3) The instrument affects the outcome only through its relation to the exposure of interest.

The first assumption means that the instrumental variable must be related, positively or negatively, to the exposure of 
interest. In addition, it is necessary that this correlation not be small. The condition that $\mathrm{Z}$ is correlated with $\mathrm{B}$ can be empirically tested, given a random sample from the population.

The second requirement is that $\mathrm{Z}$ is uncorrelated with $\varepsilon_{\mathrm{C}}$. This is an important assumption that means the instrumental variable should influence the outcome neither directly nor indirectly by its relationship with other variables. Unfortunately, whether this assumption is valid cannot be tested empirically and can be determined only on the basis of theoretic considerations.

The third requirement implies that there is no association between the instrumental variable and the outcome other than through the exposure of interest, and there are no "back-door" pathways through which the instrument can affect the outcome.

\section{INSTRUMENTAL VARIABLE ANALYSIS IN PRACTICE}

In our example, we would seek 1 or more variables that predict selection of patients into PCI after MI but do not affect the risk of mortality other than through their association with the procedure. Such a variable could be physician preference toward treatment. For example, it is conceivable that some cardiologists have a preference for pharmacologic treatment of patients with MI, whereas other cardiologists, such as interventional cardiologists, have a preference for PCI. It is also conceivable that patients' choice of their cardiovascular care provider (a clinical cardiologist or an interventional cardiologist) would be independent of their outcome (especially if referred to them by their primary care provider). Yet, patients who choose a clinical cardiologist would have a higher chance of being treated pharmacologically, whereas patients who choose an interventional cardiologist would likely be recommended PCI. Therefore, patients, by their choice (or referral) of cardiovascular care provider, would select themselves into one or the other treatment. This selection process is similar to a randomization process (natural randomization) because patients' selection of the procedure would influence their outcome post-MI only through the treatment they receive.

Another valid instrumental variable could be the distance from the patient's residence to the hospital where they receive treatment. Patients treated at a hospital equipped with a cardiac catheterization laboratory would have a higher chance of receiving PCI than those treated at a hospital without a cardiac catheterization laboratory. At the same time, the choice of the hospital would be largely dictated by distance and travel time and could be independent of the patient's outcome. Therefore, the distance from the hospital behaves similarly to a randomizer of patients into one procedure or the other.
The choice of the instrumental variable is not simple, and a bad choice leads to bad results. The validity of the instrumental variable(s) is dictated by the 3 fundamental assumptions. These assumptions are not always testable and do not always hold. A weak instrument such as one for which the correlation with the exposure is not strong would be prone to sources of error. Likewise, an instrument that is correlated directly with the outcome would not work. In our example, this would happen if interventional cardiologists were more skilled than clinical cardiologists, and as a result their patients would do systematically better than patients treated (pharmacologically) by clinical cardiologists.

\section{DISCUSSION}

Random assignment to treatment groups is a major feature of randomized controlled trials. The critical objective of randomization is to minimize systematic differences between treatment groups in the distribution of observed and unobserved pretreatment characteristics. In nonrandomized trials, the decision to indicate a treatment for a particular patient is inextricably connected to his/her clinical status and likelihood of response. In research settings where treatment is not assigned through a random mechanism, strong biases are likely to operate, whereby people who receive a particular treatment are systematically different from people who are not treated at all or treated with an alternative intervention. In situations in which treatment or exposure groups may not be comparable, an instrumental variable analysis could allow for an unbiased estimate of treatment effect. These methods work extremely well provided with a correct choice of the instrument. Of note, an instrumental variable analysis may fail if the instrument does not meet the appropriate conditions. Most commonly, a weak correlation between the instrumental variable and the exposure (weak instrument) can have significant implications for the estimate of the effect of the intervention. Unfortunately, considerable confounding by indication exists in many surgical studies, which usually weakens the correlation between the instrument and the exposure. The most valid instrumental variable is, of course, a treatment randomization scheme.

Propensity score methods are also used to reduce or eliminate the effect of treatment-selection bias when estimating treatment effects using observational data. The propensity score is defined as the probability of receiving the treatment (compared with the control exposure) conditional on a subject's observed baseline covariates. ${ }^{1}$ Therefore, the propensity score is in principle a balancing score, conditional on which treated and untreated subjects with the same propensity score will have equal distribution of measured baseline covariates. A major limitation of the propensity score method is that adjustment can be made only for observed variables 
(or confounders). In contrast, an instrumental variable analysis has the potential to adjust for all confounders, whether observed or not.

Although we have presented methods for linear regression in this article, extensions of these methods to binary outcomes and survival outcomes are well documented. ${ }^{2,3}$ Applications to problems of measurement errors and noncompliance in clinical trials are also available in the literature. ${ }^{4-6}$

\section{CONCLUSIONS}

In nonrandomized studies, the exposure groups of interest may not be comparable, and an analysis that ignores the confounding effect that unobserved or unmeasured variables have on the outcome may be severely biased. Mere acknowledgement of the bias is not sufficient to warrant publication because results of uncorrected analyses could be highly misleading. The use of instrument variables represents a valid and robust approach to the analysis of these data.

\section{Conflict of Interest Statement}

Authors have nothing to disclose with regard to commercial support.

\section{References}

1. D’Agostino R. Tutorial in biostatistics: propensity score methods for bias reduction in the comparison of a treatment to a non-randomized control group. Stat Med. 1998; 17:2265-81.

2. Rassen JA, Schneeweiss S, Glynn RJ, Mittleman MA, Brookhart MA. Instrumental variable analysis for estimation of treatment effects with dichotomous outcomes. Am J Epidemiol. 2009;169:273-84.

3. Tchetgen Tchetgen EJ, Walter S, Vansteelandt S, Martinussen T, Glymour M. Instrumental variable estimation in a survival context. Epidemiology. 2015;26: 402-10.

4. Angrist J. Estimation of limited-dependent variable models with dummy endogenous regressors: simple strategies for empirical practice. J Bus Econ Stat. 2001;19: 2-28.

5. Greenland S. An introduction to instrumental variables for epidemiologists. Int J Epidemiol. 2000;29:722-9.

6. Ye C, Beyene J, Browne G, Thabane L. Estimating treatment effects in randomised controlled trials with non-compliance: a simulation study. BMJ Open. 2014;4: e005362.

Key Words: statistical analysis, instrumental variable, confounding, bias 